Open Access

\title{
In Current Scenario various Etiological and Risk Factors those induce Polycystic Ovarian Syndrome: A Brief Review
}

\author{
G Venkat Nagaraju ${ }^{1 \star}$ and Naga Subrahmanyam $S^{2}$ \\ ${ }^{\prime}$ Department of Pharmacy Practice, Avanthi Institute of Pharmaceutical Sciences, Tagarapuvalasa, Bhogapuram, Vizianagaram, \\ Andhra Pradesh, India \\ ${ }^{2}$ Department of Pharmacy Practice, Koringa College of Pharmacy, Kakinada, Andhra Pradesh, India
}

\section{Article Info

\author{
*Corresponding author: \\ G Venkat Nagaraju \\ Assistant Professor \\ Department of Pharmacy Practice \\ Sciences \\ Tagarapuvalasa, Bhogapuram \\ Vizianagaram, Andhra Pradesh \\ India
} \\ Avanthi Institute of Pharmaceutical \\ E-mail: drnagaraju.gv@gmail.com}

Received: May 4, 2019

Accepted: May 20, 2019

Published: May 27, 2019

Citation: Nagaraju GV, Naga Subrahmanyam S. In Current Scenario various Etiological and Risk Factors those induce Polycystic Ovarian Syndrome: A Brief Review. Madridge J Immunol. 2019; 3(2): 93-94.

doi: $10.18689 / \mathrm{mjim}-1000121$

Copyright: (c) 2019 The Author(s). This work is licensed under a Creative Commons Attribution 4.0 International License, which permits unrestricted use, distribution, and reproduction in any medium, provided the original work is properly cited.

Published by Madridge Publishers

\begin{abstract}
It is one of the most common endocrine system disorders characterized by irregular menses, hyper androgenism, and polycystic ovaries that affect women in their reproductive age. Family history of PCOD may have getting the chance about $50 \%$, followed by insulin resistance and inflammation has all been linked to excess release of androgen production. But now a day's most of the girls and women addict to eat junk foods like fried food items and that food doesn't have a good quality. PCOD may induces the other health related problems like anxiety, depression, irregular menses, insulin resistance, sleep apnea, and high blood pressure.
\end{abstract}

Keywords: Poly Cystic Ovarian Syndrome; Reproductive system; Family History; Inflammation; Junk Foods; Psychiatry Diseases.

\section{Introduction}

Polycystic Ovarian Syndrome (PCOS) also termed as hyper androgenic anovulation $(\mathrm{HA})$, or Stein-Leventhal syndrome. It is one of the most common endocrine system disorders characterized by irregular menses, hyper androgenism, and polycystic ovaries that affect women in their reproductive age and it is described since 1935 by Stein and Leventhal (1935), in which the condition represents an estimate of 10 small cysts of a diameter ranging between 2 and $9 \mathrm{~mm}$ develop on one or both ovaries and/ or the ovarian volume in at least one ovary exceeds $10 \mathrm{ml}$. Systematic screening of women according to the National Institutes of Health (NIH) diagnostic criteria estimated that $4-10 \%$ of women of reproductive age suffer from PCOS. Although it was previously considered as a disorder of adult women, recent evidence suggests that PCOS is a lifelong syndrome, manifesting since prenatal age. In fact, according to the Rotterdam diagnostic criteria, the prevalence of PCOS in adolescents varies between a minimum of $3 \%$ and a maximum of $26 \%$. However, the prevalence of the disease in children is still considered unknown. The economic burden of PCOS is significantly huge manifesting since prenatal age. In fact, according to the Rotterdam diagnostic criteria, the prevalence of PCOS in adolescents varies between a minimum of $3 \%$ and a maximum of $26 \%$. However, the prevalence of the disease in children is still considered unknown. The economic burden of PCOS is significantly huge. Around 4 billion dollars are spent annually in the United States to screen for the disease and treat its various morbidities, including hirsutism, infertility, and diabetes mellitus. The Australian Health System spends more than 800 million dollars every year to account for the disease. Patients with PCOS are twice more likely to be admitted to hospital in 
comparison to patients without it [1]. Therefore, accurate and early diagnosis of PCOS is necessary not only to prevent future health co-morbidities but also to reduce financial cost and burden. In this review, we will summarize the most relevant and recent reports related to PCOS, briefly addressing the pathophysiology of the disease, then dwelling in more depth into its diagnostic criteria and their limitations in adolescence. Moreover, we will discuss morbidities associated with the classic form of PCOS and we will provide information about the various treatment regimens and screening recommendations for women living with this condition. Throughout the review, we will emphasize the complexity of PCOS in terms of Pathophysiology, diagnosis, morbidities, and the multidisciplinary treatment approach it requires [2].

\section{Etiological Factors}

- Family History of PCOD may have getting the chance about 50\% (Genes may carry PCOD diseases).

- Insulin resistance.

- Inflammation has all been linked to excess release of androgen production.

\section{Additional Risk Factors}

Now-a-day's most of the girls and women addict to eat junk foods like fried food items and that food doesn't have a good quality. So in the department of gynaecology some of the cases have to report in the KIMS Medical College, Amalapuram. As a Clinical pharmacist, I can do the interview of the patients those who are positively diagnosed with PCOD or PCOS. Some of the patients share those daily habits and life styles. In that most of the patients tell that people eat junk foods daily at evening time. So daily taking junk foods also induces the PCOD.

\section{Consequences}

Most of the Women with PCOS have a more chances for developing several serious health conditions, including lifethreatening diseases. Recent studies found that

- More than 50 percent of women with PCOS will have diabetes or pre-diabetes (impaired glucose tolerance) before the age of 40 .

- The risk of heart attack is 4 to 7 times higher in women with PCOS than women of the same age without PCOS.

- Women with PCOS are at greater risk of having high blood pressure.

- Women with PCOS have high levels of LDL (bad) cholesterol and low levels of HDL (good) cholesterol.

- Women with PCOS can develop sleep apnea. This is when breathing stops for short periods of time during sleep.

- Women with PCOS may also develop anxiety and depression.
- Women with PCOS are also at risk for endometrial cancer. Irregular menstrual periods and the lack of ovulation cause women to produce the hormone oestrogen, but not the hormone progesterone. Progesterone causes the endometrium (lining of the womb) to shed each month as a menstrual period. Without progesterone, the endometrium becomes thick, which can cause heavy or irregular bleeding. Over time, this can lead to endometrial hyperplasia, when the lining grows too much, and cancer develops [3].

\section{Discussion and Conclusion}

Finally, we concluded that most are the PCOD positive patients are having a family history of PCOD, getting the chance about $50 \%$, followed by insulin resistance and inflammation has all been linked to excess release of androgen production. But now a day's most of the girls and women are addicted to eating junk foods like fried food items and that food doesn't have good quality. As a Clinical pharmacist, I have done the interview of the patients those who were positively diagnosed with PCOD or PCOS. Some of the patients share those daily habits and lifestyles. In that most of the patients tell that people eat junk foods daily at evening time. So daily taking junk foods also induces the PCOD, in sometimes PCOD may induces other health related problems like anxiety, depression, irregular menses, insulin resistance, sleep apnea, and high blood pressure.

\section{References}

1. Açbay $O$, Gündogdu S. Can metformin reduce insulin resistance in polycystic ovary syndrome? Fertil Steril. 1996; 65(5): 946-949.

2. Anderson H, Fogel N, Grebe SK, Singh RJ, Taylor RL, Dunaif A. Infants of women with polycystic ovary syndrome have lower cord Blood and rostenedione and estradiol levels. J Clin Endocrinol Metab. 2010; 95: 2180-2186. doi: 10.1210/Jc.2009-2651

3. PCOS Awareness association. PCOS Overview. 2017. 